\title{
A Novel Grid-Wide Transient Stability Assessment and Visualization Method for Increasing Situation Awareness of Control Room Operators
}

\author{
Pertl, Michael; Rezkalla, Michel M.N.; Marinelli, Mattia
}

Published in:

2016 IEEE PES Innovative Smart Grid Technologies Asia

Link to article, DOI:

10.1109/ISGT-Asia.2016.7796366

Publication date:

2016

Document Version

Peer reviewed version

Link back to DTU Orbit

Citation (APA):

Pertl, M., Rezkalla, M. M. N., \& Marinelli, M. (2016). A Novel Grid-Wide Transient Stability Assessment and Visualization Method for Increasing Situation Awareness of Control Room Operators. In 2016 IEEE PES Innovative Smart Grid Technologies Asia (pp. 87-92). IEEE. https://doi.org/10.1109/ISGT-Asia.2016.7796366

\section{General rights}

Copyright and moral rights for the publications made accessible in the public portal are retained by the authors and/or other copyright owners and it is a condition of accessing publications that users recognise and abide by the legal requirements associated with these rights.

- Users may download and print one copy of any publication from the public portal for the purpose of private study or research.

- You may not further distribute the material or use it for any profit-making activity or commercial gain

- You may freely distribute the URL identifying the publication in the public portal 


\title{
A Novel Grid-Wide Transient Stability Assessment and Visualization Method for Increasing Situation Awareness of Control Room Operators
}

\author{
Michael Pertl, Michel Rezkalla, Mattia Marinelli \\ Center for Electric Power and Energy, Department of Electrical Engineering \\ Technical University of Denmark (DTU) \\ Contact: mpertl@elektro.dtu.dk
}

\begin{abstract}
The aim of the paper is to introduce a grid-wide assessment method to determine the transient stability margin and visualize it effectively to increase the situation awareness of control room operators. Critical area(s) with insufficient transient stability margin have to be identified in order to be able to take appropriate preventive actions. The introduced method evaluates the transient stability margin with a time-domain approach by using the voltage angle of several buses across the power system. Information about the severity of a contingency and the location of the most critical buses is derived. Moreover, it is shown that the method facilitates the visual examination of transient stability. It provides control room operators with essential information about the state of the system and enables them to take appropriate preventive actions if insufficient transient stability margins are detected.
\end{abstract}

Index Terms-Control Room Operator, Converter Based Generation, Situation Awareness, Time Domain Simulation, Transient Stability

\section{INTRODUCTION}

As the penetration of renewable energy sources (RES) is increasing, their impact on the dynamic behavior of power systems may no longer be neglected. The integration of RES results in substantial changes to the power system characteristics due to changed load flow patterns, commitment and dispatch of existing synchronous generators (SGs). New components with different dynamic behaviors, such as wind and solar generation, are introduced [1]. Moreover, the inertia of power systems varies in the course of time depending on the energy mix and cannot be assumed constant anymore. Predominantly, type three doubly-fed induction generators (DFIG) and type four fully-rated power converter wind turbines are being installed due to their good controllability compared to directly grid-connected wind turbines of type one and two [2]. Similar to type four wind power plants, solar generation is connected to the power grid through a fully-rated power converter and, thus, it is completely decoupled from the grid, i.e. no inertia is provided to the grid.

The paradigm shift from conventional SGs to converter based generation affects the transient stability of power systems. Transient stability is a very important property of power systems since instability can lead to widespread outages due to the tripping of synchronous generators (SG). Therefore, researchers focused on investigating the characteristics and transient behavior of RES-components that are being installed in the power system on a device-by-device basis [3]-[9]. Going one step further, an analysis of the RES integration impact on the grid-wide transient stability of power systems has to be carried out as this issue has barely been addressed yet [3], [10], [11].

Transient stability is the ability of a power system to maintain synchronism after being subject to a large disturbance (e.g. three-phase fault) and is categorized under short term rotor angle stability according to [12]. Obviously, a large disturbance will not only affect the rotor angle displacement of synchronous generators but it will also affect other system variables, such as voltage magnitudes and frequency. In fact, transient stability and short term voltage stability are closely linked. A progressive drop in bus voltages can also be associated with rotor angle instability, e.g. when the rotor angles between two groups of machines approaches $180^{\circ}$ it causes a rapid drop in voltages at intermediate points in the network close to the electrical center [12]-[14]. In the past, the equal area criterion, the single machine equivalent method, Lyapunov's direct approaches and critical fault clearing time (CCT) approach were usually used to assess the transient stability. Since these methods are limited to SGs, they cannot be applied to converter based generation units which may also induce active power swings provoking rapid voltage drops. Hence, a method which allows to assess the transient stability of SGs and possible active power swings of converter based units has to be developed.

This paper focuses on the development of a novel gridwide transient stability assessment and visualization method for increasing situation awareness of control room operators. The method also enables a comparison of the transient stability of a power system with high share of RES under different operating conditions. Furthermore, the effectiveness of preventive actions which may be applied to the system can be assessed.

Control room operators are faced with a power system's growing complexity, size and its various new components and technologies. Therefore, the situation awareness of control room operators about the current system state and the anticipation of future problems is becoming increasingly important but also more challenging [15]. A great share of situation awareness problems are related to lack of information, i.e. the 
information that is needed is not provided [16]. The method presented in this paper aims at providing additional synthetic information about the state of the system in terms of transient stability without overloading the control room operator.

\section{METHOD}

\section{A. Transient Stability Approach}

The introduced method includes assessment and visualization of transient stability on a system-wide basis. It is also able to capture active power swings induced by converter based generation units. A time domain (TD) approach is used to assess the transient stability. Opposed to traditional methods, voltage angles at the buses are used to determine whether the grid in its current operational state can sustain a particular large disturbance. The justification why voltage angles at the buses instead of rotor angles are used to examine the transient stability is explained by means of the two-machine system in Fig. 1. The rotor angle between the two machines $\varphi_{G M}$ is determined by the power transfer, the sum of the reactances between them and the two internal machine voltages. This highly nonlinear relationship, given in (1), is valid for high voltage grids due to the high $X / R$ ratio, but obviously it will deviate significantly for multi-machine systems considering automatic voltage regulators and other effects. However, the general form is similar. The vector diagram of the two-machine system in Fig. 2 shows that the internal generator voltage is leading the internal motor voltage by the angle $\varphi_{G M}$. A greater power transfer leads to an increased angle displacement. Consequently, also the angle $\varphi_{L}$ between the two buses increases which enables an assessment of transient stability by utilizing the voltage angles. When the angle between two machines or groups of machines reaches $180^{\circ}$ during transient conditions, a loss of synchronism is most likely. That will cause large fluctuations in voltage, current and consequently in the power output of the generator, which eventually leads to voltage angle swings in the system. Additionally, rapid voltage drops at the intermediate point in the network could occur due to the large angle displacement. Since there is no rotor angle in converter based generation, the voltage angle at their buses can be used to evaluate the transient stability on a grid-wide basis. The voltage angle displacement of the bus voltages will indicate whether the power system can sustain a particular contingency without loosing synchronism and furthermore indicate if large power swings of converter based generation units occur.

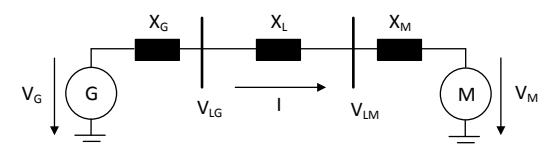

Fig. 1. Two-machine system

$$
P=\frac{V_{G} V_{M}}{X_{T}} \sin \left(\varphi_{G M}\right)
$$

with

$$
X_{T}=X_{G}+X_{L}+X_{M}
$$

When a generator falls out-of-step, it has a pole slip which means the rotor angle displacement between the critical generator and the other generators in the system exceeds $180^{\circ}$ which will also be reflected in the voltage angle at the generator bus. If no pole slip occurs, the rotor angle displacement does not exceed the critical limit of $180^{\circ}$ following that the voltage angles at the buses neither exceeds this limit. Unfortunately, no fixed limit for the maximum voltage angle displacement can be set which guarantees that the rotor angles are not reaching the critical limit but it is suggested that the maximum voltage angle displacement should not exceed $120^{\circ}$ to maintain some security headroom.

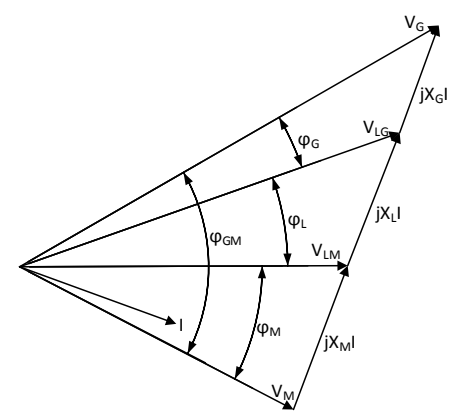

Fig. 2. Vector diagram for the two-machine system

In the following paragraphs, the proposed procedure of the transient stability approach is explained in detail. A flow chart of the approach is shown in Fig. 3 where the red frame highlights the focus of this work. Parts outside the red frame are not covered in particular, but mentioned due to their importance for the approach.

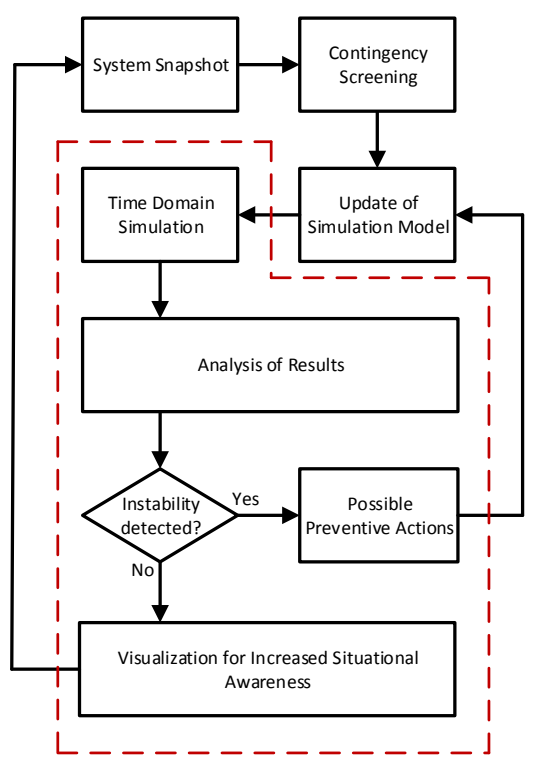

Fig. 3. Flow chart of the transient stability approach

System snapshot: Two possibilities to obtain a snapshot of the state of the system. The data about the current state of the grid is extracted from SCADA system. The data includes 
breaker status, generation dispatch, activation of capacitor banks and further relevant data for the assessment. The second way of getting a system snapshot is to derive the system state from measurements of phasor measurement units (PMU) which assumes full power system observability by PMUs.

Contingency screening: To assess the transient stability a contingency has to be applied in the TD simulation. Theoretically, every potential contingency which could perturb the system has to be analyzed to guarantee transient stability. Certainly, it is not possible to include every possible contingency in the assessment since a TD approach is used and computational effort should be kept within reasonable limits. Therefore, a contingency screening to determine the most critical buses with regard to electrical faults is needed. Within this work only one three-phase fault was used since the contingency screening is beyond the scope of this paper.

Update of simulation model: The simulation model which represents the real system has to be updated to comply with current system state before starting the TD simulation. Generator dispatch, breaker status, activated capacitor banks and generation from RES have to be refreshed.

Time domain simulation: Time domain simulations are carried out for the most severe contingencies determined in the preceding screening. The duration of the simulation is flexible, but the computational effort grows with increasing simulation time. A fixed simulation time up to a few seconds depending on available computational power and security level should be set to capture first swing instabilities. An early stop of the assessment when detecting an instability on the fly could be implemented to reduce the computational power even more.

Analysis of the results: In the TD simulation the voltage angles $\varphi_{U, B u s_{i}}$ are referenced to the voltage angle of the slack bus. In order to make the voltage angle calculation independent from the slack bus definition, the average voltage angle $\varphi_{U, \text { avg }}$ is calculated to serve as an artificial reference, similarly to the definition of the center of inertia. Then the voltage angles $\varphi_{U, B u s_{i}, \text { nref }}$ are calculated with the new reference according to (3).

$$
\varphi_{U, B u s_{i}, n r e f}=\varphi_{U, B u s_{i}}-\varphi_{U, a v g}
$$

Following the calculation of the slack-independent voltage angle, the absolute maximum of the voltage angle for every bus is determined. The concept is visualized in Fig 4. The dashed lines are the original voltage angle results from the TD simulation which are referenced to the slack bus. The black line represents the calculated average of the original voltage angles. The solid lines are the calculated voltage angles with reference to the average.

Instability detection: If voltage angles exceed $120^{\circ}$, an instability is detected. Since voltage angles are used rather than the rotor angles the limit for transient stability is set to this lower value.

Possible preventive action: Available counteractions highly depend on the properties of the particular power system. The following counteractions are of interest from an operational perspective: Installed capacitor banks, demand response, load

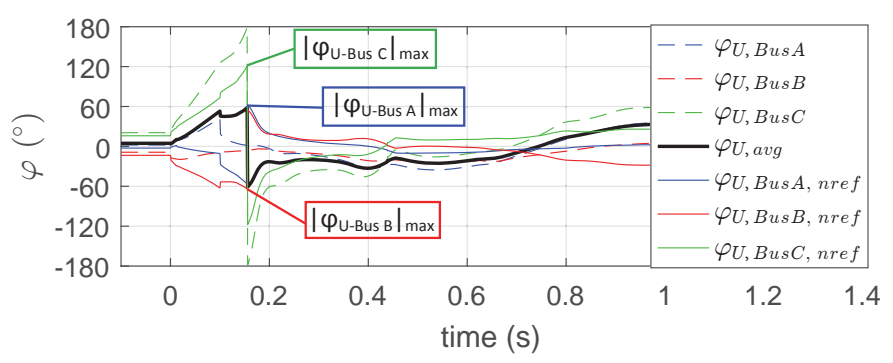

Fig. 4. Illustration of the concept to obtain the slack-independent maximum absolute voltage angle deviation for three buses

shedding capability, generation re-dispatch possibility and temporary increase of voltage setpoints.

Visualization for increased situation awareness: The visualization is one of the key points of the procedure. It has to be informative by highlighting the most important information which should be communicated to the control room operator. The results are visualized as bar plot with the bus notation on the $\mathrm{x}$-axis, scenario number on the $\mathrm{y}$-axis and the height of the bars is determined by the maximum absolute voltage angle, see Fig. 6 and 7. The bars are colored according to their height, i.e. the highest bars are colored red and the lowest are colored blue. This type of coloring eases the recognition of the highest deviations across the power system. The highest deviations are seen at the first glance.

\section{B. Reference Grid and Modeling Considerations}

The assessment and visualization approach is tested by means of a $\mathrm{HV}$ reference grid developed in the currently running EU project ELECTRA (electrairp.eu) and is shown in Fig. 5. It has been developed to serve as a future HV benchmark grid for RES integration studies. Detailed information about the Pan-European simulation model is published in [17]. The simulation model was developed in the power system simulation software DIgSILENT PowerFactory.

In order to achieve realistic results with TD simulations, some important considerations have to be made prior to the analysis. This includes, but is not limited to, the models of (a)synchronous machines, transformers, converters and loads. Special attention has to be paid to load modeling due to its large impact on the results. The loads are representing the aggregated behavior of the underlying distribution system. Thus, the loads are modeled as voltage and frequency dependent according to the ZIP ( $\mathrm{Z}=$ const. impedance, $\mathrm{I}=$ const. current, $\mathrm{P}=$ const. power) approach analytically described in (4) and (5). However, the load characteristics have to be adjusted according to the load distribution of the considered system. In the absence of sufficient data about the load distribution, the coefficients for voltage and frequency dependence can be set according to the common practice for stability studies which is recommended in [18]. Constant current $(\alpha=1)$ can be assumed for active power and constant impedance $(\beta=2)$ for reactive power sensitivity. This applies for voltages between 0.7 and $1.2 \mathrm{pu}$ and is changed to constant impedance 
if voltage exceeds this limits to avoid computational problems. Frequency dependence is assumed to be $K_{p f}=1.5$ and $K_{q f}=-1$ for active and reactive power respectively. The parameters for the load models are summarized in Table I.

$$
\begin{aligned}
& P=P_{0} \cdot\left(\bar{V}^{\alpha}\right)\left(1+K_{p f} \Delta f\right) \\
& Q=Q_{0} \cdot\left(\bar{V}^{\beta}\right)\left(1+K_{q f} \Delta f\right)
\end{aligned}
$$

with

$$
\bar{V}=\frac{V}{V_{0}} \quad \text { and } \quad \Delta f=f-f_{0}
$$

$P, Q$ - actual active/reactive power drawn by the load

$P_{0}, Q_{0}$ - nominal active/reactive power of the load

$V$ - actual voltage

$V_{0}$ - nominal voltage

$\alpha, \beta$ - exponential coefficients for characterization of ZIP

( $0=$ const. power, $1=$ const. current, $2=$ const. impedance $)$

$K_{p f}, K_{q f}$ - linear coefficients for frequency dependence of active and reactive power respectively

TABLE I

SuMMARY OF LOAD MODEl PARAMETERS

\begin{tabular}{|c|c|c|c|}
\hline \multicolumn{2}{|c|}{ Voltage Dependence } & \multicolumn{2}{c|}{ Frequency Dependence } \\
\hline \hline$\alpha=\partial P / \partial V$ & $\beta=\partial Q / \partial V$ & $K_{p f}=\partial P / \partial f$ & $K_{q f}=\partial Q / \partial f$ \\
\hline 1 & 2 & 1.5 & -1 \\
\hline
\end{tabular}

The simulation model comprises four cells [19] with different properties in terms of generation portfolio, load and voltage level/type. The total load of the system corresponds to $2600 M W$ with $\cos (\varphi)=0.95$ (ind). The synchronous generators in cell one and two represent gas turbines while the synchronous generators in cell three are hydraulic turbines. All generators are equipped with the IEEET1 excitation system which is available as a standard model in the PowerFactory software [20]. The multi-terminal DC (MT-DC) grid in cell four connects a static generation unit with the $\mathrm{AC}$ grid at four different locations via voltage source converters (VSC).

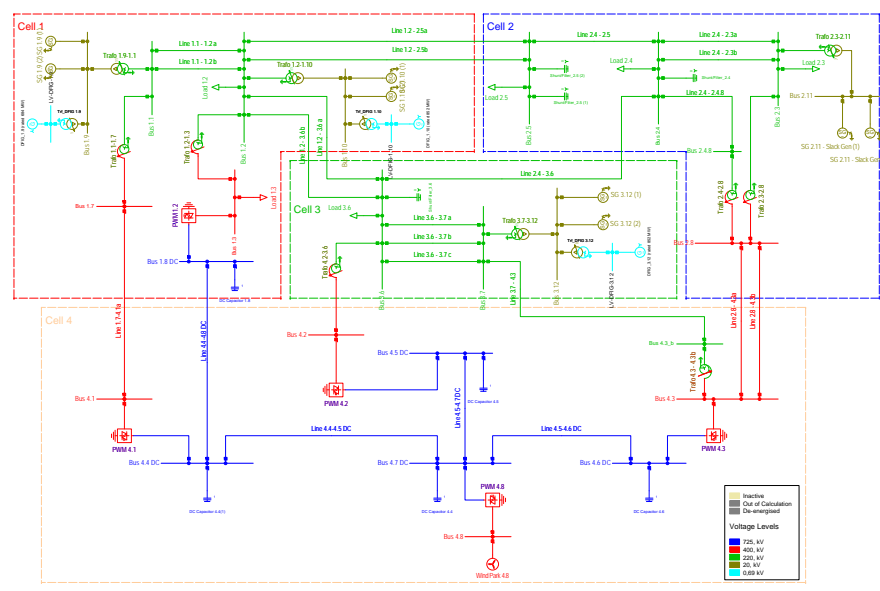

Fig. 5. Pan-European simulation model
The VSCs are controlled according to a master/slave principle. Converter 4.3 controls the $\mathrm{AC}$ and the $\mathrm{DC}$ voltage at its terminals and thus acts as the master. The other grid connected converters control their AC terminal voltage and the active power flow and are hence called slaves. Converter 4.8 connects the static generation unit with the MT-DC grid and controls the $\mathrm{AC}$ voltage and frequency at the remote AC Bus 4.8. The static generation unit can represent any kind of converter connected generation.

The DFIG models are originating from the PowerFactory library and are available as templates with rated powers from $1-6 M W$. The $6 M W$-type with 65 parallel units was used in the analysis. This configuration of wind parks with 65 . $6 M W$ units is installed at three different locations which correspondents to a total installed capacity of $1170 \mathrm{MW}$ (3 . $65 \cdot 6 \mathrm{MW}$ ) DFIG generation.

\section{Simulation Scenarios}

To analyze the transient stability, four simulation scenarios with different levels of RES penetration have been assumed. The generation from RES ranges from 0 to $75 \%$ with respect to the total load. To set up particularly challenging simulation scenarios, a heavily loaded system was assumed since generally the transient stability of highly loaded systems is lower than in lightly loaded ones. This is mainly caused by operating SGs closer to their limits, i.e. smaller stability margin [10]. Table II summarizes the key aspects of the four simulation scenarios, such as RES penetration level $P_{R E S}$ in relative and absolute values, number and setpoint of active synchronous generators and the system inertia $2 H$. The share of RES generation is split into $50 \%$ of DFIG and $50 \%$ of full converter generation.

TABLE II

Simulation SCENARIOS

\begin{tabular}{|c||c|c|c|c|c|}
\hline $\begin{array}{c}\text { Scenario } \\
\text { No. }\end{array}$ & $\begin{array}{c}\mathbf{P}_{\text {RES,rel }} \\
(\boldsymbol{\%})\end{array}$ & $\begin{array}{c}\mathbf{P}_{\text {RES }} \\
(\mathbf{M W})\end{array}$ & $\begin{array}{c}\text { No. of SG } \\
\text { in service }\end{array}$ & $\begin{array}{c}\text { Pset of } \\
\text { SGs } \\
(\mathbf{M W})\end{array}$ & $\begin{array}{c}\text { System } \\
\text { inertia } \mathbf{2 H} \\
(\mathbf{s})\end{array}$ \\
\hline \hline 1 & 0 & 0 & 8 & 325 & 10 \\
\hline 2 & 25 & 650 & 6 & 325 & 7.5 \\
\hline 3 & 50 & 1300 & 6 & 217 & 7.5 \\
\hline $4(.1)$ & 75 & 1950 & 3 & 217 & 3.75 \\
\hline
\end{tabular}

Scenario 1: Scenario one builds the base case without penetration of RES and exclusively generation from all the eight SGs.

Scenario 2: In scenario two only six generators are in service due to RES generation of $25 \%$. The setpoints of the SGs in scenario two are the same as in one due to decommissioning of two SGs.

Scenario 3: Six SGs are in service with reduced setpoint due to increase in RES generation up to $50 \%$.

Scenario 4: Scenario four represents a possible future scenario with a share of $75 \%$ of RES. Three more generators are decommissioned and only three SGs remain in service. 
Scenario 4.1: Scenario 4.1 is equal to Scenario 4 with the only difference that a counteraction for transient stability improvement is applied, namely the initial voltage setpoint of the DFIGs at Bus 1.9. is increased from 1 to $1.05 \mathrm{pu}$.

Two study cases (SC) with a three-phase short circuit at Bus 1.10 are analyzed. The fault was initiated at $t=0 \mathrm{~s}$ and cleared after $100 \mathrm{~ms}$ (SC 1) and $200 \mathrm{~ms}$ (SC 2), respectively. The fault impedance was set to $Z_{F}=(0.001+j 0.001) \Omega$.

\section{RESUlTS AND Discussion}

\section{A. Fault Analysis and Visualization}

The results obtained from the simulation are shown and discussed in this section. The main focus is the voltage angle and, therefore, frequency and voltage magnitude are not shown. At first, the results within each SC are analyzed, then the two SCs are compared. Finally, an action to improve transient stability has been applied in scenario four of SC 2 in order to make the improvement visible.

Fig. 6 shows the results of SC 1, a three-phase fault for $100 \mathrm{~ms}$ at Bus 1.10. It is clearly visible that the differences between the first three scenarios are marginal while the results of the fourth scenario differ significantly. Three significant peaks are visible with amplitudes of approximately $175^{\circ}$ and $94^{\circ}$ labeled in red and light green, respectively. Peculiarly, the maximum angle deviation of the faulty Bus 1.10 is not the largest. Instead, the Buses 1.7, 1.9 and 4.1 are highly affected. Since the maximum angle deviation is close to $180^{\circ}$ at Bus 4.1, the contingency is likely to cause a transient instability. However, no SG is connected at Bus 4.1 but a converter (PWM 4.1) which injects active power into the AC grid. The large angle excursion is caused by a large active power swing of the converter during the disturbance which is also seen at the adjacent Buses 1.7 and 1.9. This is clearly an undesired condition and has to be avoided by taking countermeasures. Noteworthy, in scenario four only three synchronous generators are connected and in the area of the buses, identified as critical, no synchronous generation is present.

Fig. 7 shows the results of SC 2, a three-phase fault for $200 \mathrm{~ms}$ at Bus 1.10. The results for the first three scenarios are similar to the results in SC 1 but, as expected, the angle

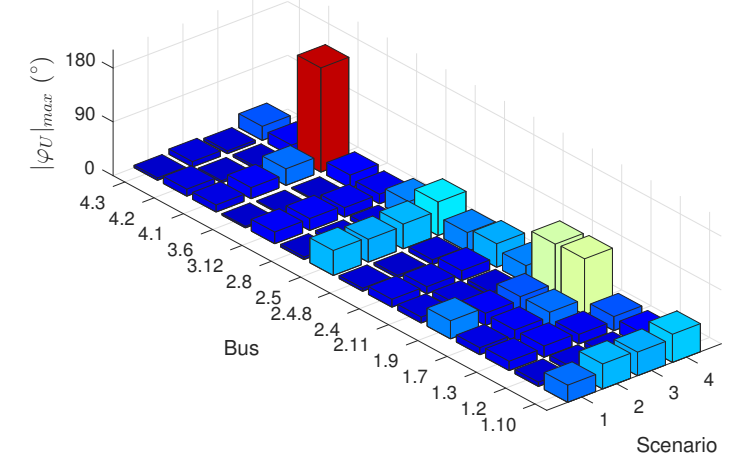

Fig. 6. Results of SC 1: Three-phase fault at Bus 1.10 for $100 \mathrm{~ms}$

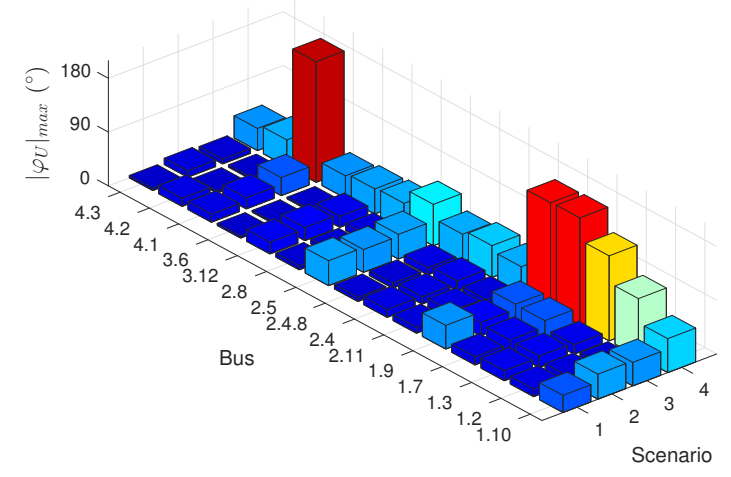

Fig. 7. Results of SC 2: Three-phase fault at Bus 1.10 for $200 \mathrm{~ms}$

deviations are slightly larger than in SC 1. The contingency shows again the largest impact in scenario four with the highest RES penetration and the lowest system inertia. Notably, the same buses are affected as in SC 1 since the location of the fault has not changed but the duration was double. That is also reflected in the results since the Buses 1.7 and 1.9 show deviations of approximately $180^{\circ}$. Bus 4.1 shows again the largest deviation of approximately $200^{\circ}$. The maximum deviations of Bus 1.2 and 1.3 are also increased compared to SC 1, i.e. the contingency has a more widespread effect and is therefore more severe.

\section{B. Preventive Actions to Ensure Transient Stability}

If instabilities are detected, appropriate preventive actions have to be taken in order to ensure transient stability. Preventive actions have to be applicable in power system operation. The control room operator must be able to apply the change to the power system. A generation re-dispatch can be very effective. Depending on the properties of the particular power system, installed capacitor banks, demand response capability and, as last resort, also load shedding are preventive actions to ensure transient stability during operation. Moreover, a temporary increase of the voltage setpoints in the area(s), identified as critical, may be a suitable counteraction. However,

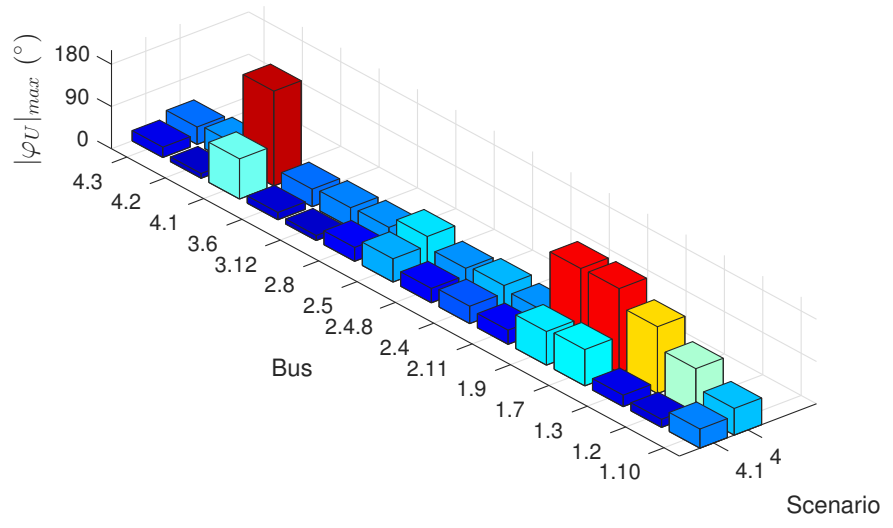

Fig. 8. Comparison of the voltage angles for SC 2: Scenario 4 and 4.1 with preventive action applied 
various factors such as technical feasibility, regulatory aspects, economical efficiency and possible adverse effects on other stability domains have to be considered when determining an appropriate counteraction.

To visualize how the counteraction affects the power system the voltage setpoint of the DFIGs in the critical area at Bus 1.9 in SC 2/Scenario 4 has been increased from $1 \mathrm{pu}$ to $1.05 \mathrm{pu}$. The results of the simulation are shown in Fig. 8 where scenario 4.1 is the scenario with the increased voltage setpoint. The improvement can be seen clearly in Fig. 8 since the maximum angle deviation of all buses has been reduced significantly.

\section{CONCLUSION AND FUture WORK}

This paper presented a novel grid-wide transient stability assessment and visualization method for increasing the situation awareness of control room operators. It eases the visual examination of transient stability issues on a grid-wide basis through the use of a coloring which facilitates the perception of the communicated information. The method enables an examination of the effect of a particular preventive action on the grid. The maximum absolute voltage angle deviation at the buses across the grid are plotted in a bar plot, with the colors set according to their height. One of the advantages of the method is that it is not dependent on the rotor angles of synchronous machines. Thus, it can also be applied under the presence of high share of converter based generation to detect large active power swings induced by converter based units. It was shown that the transient stability can be improved by increasing the voltage setpoint of DFIGs in the critical area. Moreover, the effect of the counteraction can be represented properly in the visualization.

However, control room operators are exposed to an enormous amount of information of different types. The visualization of the transient stability assessment has to be integrated into the existing SCADA or energy management system (EMS) to provide the operator with the needed information for maximum situation awareness. Work concerning the contingency screening has to be carried out. The most critical buses of the grid, where a fault would have the largest impact on the grid, have to be determined in order to reduce the computational effort needed. Moreover, the effectivity of preventive actions has to be studied in terms of technical feasibility and from economical perspective. To allow decision support for the control room operator a ranking method of the potential preventive actions has to be developed. Investigations on how RES can contribute to transient stability improvement have to be made. In addition, it has to be studied if the preventive actions are interfering with other operational objectives.

\section{ACKNOWLEDGMENT}

Michael Pertl is a PhD student at the Technical University of Denmark (DTU) and is supported by the EU FP7 project ELECTRA (grant: 609687) and the Danish Research Project ELECTRA Top-up (grant: 3594756936313). More information can be found at electrairp.eu.

\section{REFERENCES}

[1] N. W. Miller, M. Shao, S. Pajic, and R. D'Aquila, "Western Wind and Solar Integration Study Phase 3: Frequency Response and Transient Stability," tech. rep., National Renewable Energy Laboratory (NREL), Denver, 2014.

[2] M. Cheng and Y. Zhu, "The state of the art of wind energy conversion systems and technologies: A review," Energy Conversion and Management, vol. 88, pp. 332-347, 2014.

[3] J. Keller and B. Kroposki, "Understanding Fault Characteristics of Inverter-Based Distributed Energy Resources Understanding Fault Characteristics of Inverter-Based Distributed Energy Resources," Tech. Rep. January, National Renewable Energy Laboratory (NREL), Denver, 2010.

[4] M. Marinelli, S. Massucco, A. Mansoldo, and M. Norton, "Analysis of Inertial Response and Primary Power-Frequency Control Provision by Doubly Fed Induction Generator Wind Turbines in a Small Power System," in 17th Power Systems Computation Conference, (Stockholm), pp. 1-7, 2011.

[5] J. Ekanayake and N. Jenkins, "Comparison of the response of doubly fed and fixed-speed induction generator wind turbines to changes in network frequency," IEEE Transactions on Energy Conversion, vol. 19, no. 4, pp. 800-802, 2004

[6] S. Eftekharnejad, V. Vittal, G. T. Heydt, B. Keel, and J. Loehr, "Impact of Increased Penetration of Photovoltaic Generation on Power Systems," IEEE Transactions on Power Systems, vol. 28, no. 2, pp. 893-901, 2013.

[7] M. Edrah, K. L. Lo, and O. Anaya-Lara, "Impacts of High Penetration of DFIG Wind Turbines on Rotor Angle Stability of Power Systems," IEEE Transactions on Sustainable Energy, vol. 6, no. 3, pp. 759-766, 2015.

[8] E. Vittal, M. O’Malley, and A. Keane, "Rotor angle stability with high penetrations of wind generation," IEEE Transactions on Power Systems, vol. 27, no. 1, pp. 353-362, 2012.

[9] L. Meegahapola and T. Littler, "Characterisation of large disturbance rotor angle and voltage stability in interconnected power networks with distributed wind generation," IET Renewable Power Generation, vol. 9 , no. 3, pp. 272-283, 2015.

[10] C. Liu, Z. Chen, C. L. Bak, and Z. Liu, "Transient Stability Assessment of Power System with Large Amount of Wind Power Penetration : the Danish Case Study," Area, pp. 461-467, 2012.

[11] N. W. Miller, "Transient Stability in a World of Wind and Solar Generation," IEEE power \& energy magazine, pp. 31-39, nov 2015.

[12] K. Prabha, J. Paserba, V. Ajjarapu, G. Anderson, A. Bose, C. Canizares, N. Hatziargyriou, D. Hill, A. Stankovic, C. Taylor, T. Van Vutsem, and V. Vittal, "Definition and Classification of Power System Stability," IEEE Transactions on Power Systems, vol. 21, no. 3, pp. 1387-1401, 2004.

[13] P. Kundur, Power system stability and control. McGraw Hill, 1994.

[14] T. Weckesser, H. Johannsson, J. Østergaard, and T. Van Cutsem, "Derivation and application of sensitivities to assess transient voltage sags caused by rotor swings," International Journal of Electrical Power and Energy Systems, vol. 72, pp. 75-82, 2015.

[15] M. Panteli and D. S. Kirschen, "Situation awareness in power systems: Theory, challenges and applications," Electric Power Systems Research, vol. 122, pp. 140-151, 2015.

[16] M. Endsley, "Situation Awareness in the Bulk Power System," in Human Performance Conference, (Atlanta), 2012.

[17] M. Marinelli, M. Pertl, M. Rezkalla, M. Kosmecki, S. Canavese, A. Obushevs, and A. Morch, "The Pan-European Reference Grid Developed in the ELECTRA Project for Deriving Innovative Observability Concepts in the Web-of-Cells Framework," in 51th International UniversitiesPower Engineering Conference (UPEC), (Coimbra), pp. 1-6, 2016, submitted.

[18] K. Sun, "Power System Operations \& Planning - Load Modeling," 2015.

[19] L. Martini, L. Radaelli, H. Brunner, C. Caerts, A. Morch, S. Hänninen, and C. Tornelli, "ELECTRA IRP Approach to Voltage and Frequency Control for Future Power Systems with High DER Penetration," in 23rd International Conference on Electricity Distribution (CIRED), (Lyon), pp. 1-5, 2015.

[20] DIgSILENT, "Standard Models in PowerFactory," User Manual - Appendix D, pp. 1097-1117, 2015. 